\title{
Relación entre Antecedente Empresarial Familiar y el Emprendimiento de Micro y Pequeñas Empresas
}

\author{
Irma Leticia Chávez-Márquez (PhD) \\ Liliana Álvarez-Loya (MARH) \\ Blanca Margarita Rosas-García (MARH) \\ Luis Raúl Sánchez-Acosta (MF) \\ Universidad Autónoma de Chihuahua, México
}

Doi: 10.19044/esj.2017.v13n28p81 URL:http://dx.doi.org/10.19044/esj.2017.v13n28p81

\begin{abstract}
The objective of the research was to determine the relationship between the family business background and the entrepreneurship of micro and small enterprises. A total census of 123 companies registered in the Business Incubator of the Facultad de Contaduría y Administración of the Universidad Autónoma de Chihuahua in Chihuahua, Mexico, was carried out. The research was descriptive and exploratory; using the analyticalsynthetic method, with a transectional design, performed between April and June 2016. The dependent variable was the generation of companies and the independent variable was to have a family business background. The main results indicate that the majority of entrepreneurs with companies in operation, have a family business background. However, it is not decisive to not have a relative with a company, and other factors can also influence the entrepreneurship of new companies, among which may be the support provided by the business incubator.
\end{abstract}

Keywords: Micro and small enterprises, business incubator, companies in operation

\section{Resumen}

El objetivo de la investigación fue determinar la relación entre el antecedente empresarial familiar y el emprendimiento de micro y pequeñas empresas. Se realizó un censo total de 123 empresas registradas en la Incubadora de Negocios de la Facultad de Contaduría y Administración de la Universidad Autónoma de Chihuahua, en Chihuahua, México. La investigación fue descriptiva y exploratoria; utilizando el método analíticosintético, con un diseño transeccional, realizado entre los meses de abril a junio de 2016. La variable dependiente fue la generación de empresas y la 
variable independiente fue contar con un antecedente empresarial familiar. Los principales resultados indican que la mayoría de los emprendedores con empresas en operación, cuentan con un antecedente familiar empresarial. Sin embargo, no es determinante el hecho de no tener un familiar con una empresa, pudiendo influir también otros factores en el emprendimiento de nuevas empresas, entre los cuales puede estar el apoyo brindado por la incubadora de negocios.

Palabras-clave: Micro y pequeñas empresas, incubadora de negocios, empresas en operación

\section{Introducción}

El desarrollo económico generado en un país es determinado en gran medida por la creación de nuevas empresas, las cuales serán generadoras de una serie de beneficios, entre los cuales se encuentran aumento en la productividad y generación de empleos. El ser emprendedor en una edad temprana, presenta ventajas en la creación de empresas, debido al espíritu innovador pujante por naturaleza de los jóvenes.

Dadas las características del entorno sociodemográfico en donde es necesario generar oportunidades para el desarrollo de los jóvenes, en la Facultad de Contaduría y Administración (FCA) de la Universidad Autónoma de Chihuahua (UACH) resulta imperativo el impulsar un espíritu emprendedor de la mano de una capacidad para el desarrollo de empresas, para lo cual se desarrollaron una serie de acciones y estrategias. Entre las estrategia generadas, se creó la Incubadora de negocios, la cual brinda atención a todos aquellos emprendedores que deseen poner en marcha una empresa; donde el apoyo se brinda tanto a alumnos como a la comunidad en general.

El entorno familiar podría ser un factor detonante en la creación de nuevas empresas, porque puede ir ligado con el hecho de tener algún antecedente familiar empresarial, con el cual se está familiarizado, teniendo de primera mano la experiencia generada al vivir cercanamente la dinámica de los entornos empresariales.

Dentro de nuestra sociedad, se considera a la familia como un factor fundamental para la formación de individuos que contribuyan con su comunidad, con actitudes y actividades responsables, como parte del desarrollo económico de su entorno entre otros. Existen en ocasiones situaciones laborales negativas de algún integrante de la familia, en donde esta interviene para la generación de nuevas oportunidades.

Una institución de gran importancia económica es la empresa, como célula básica del sistema de generación de bienes y servicios, permite la manifestación de la creatividad y la libertad jurídica de las personas. La 
empresa aporta entre otros, el trabajo y otros más, aportan sus habilidades de dirección, para conseguir determinados fines económicos. Actualmente las tendencias mundiales en el campo empresarial reconocen en las MIPYMES una parte fundamental de la economía de cualquier nación. Más del 90\% del universo de empresas formales e informales en los distintos países se ubican en este grupo, participando también de forma muy elevada en las ventas totales, las exportaciones, el PIB y el empleo. Cualquier dificultad general en estas organizaciones de pequeño tamaño repercute de manera negativa en los indicadores macroeconómicos y sociales de cualquier país. (Valdés \& Sánchez, 2012)

El crecimiento acelerado de pequeñas empresas se puede dar en economías muy dinámicas y con gran capacidad emprendedora, que en épocas de auge económico permiten el desarrollo de nuevas empresas mediante el aprovechamiento de los nuevos nichos de mercado y la disponibilidad del talento empresarial; pero también en épocas de recesión económica la capacidad emprendedora se pone a prueba para dar respuesta, haciendo acopio de la creatividad, a nuevas opciones empresariales capaces de iniciarse en mercados deprimidos. En los dos casos, es necesario contar con una política pública que fomente el desarrollo empresarial de manera incluyente; es decir, que por una parte premie la capacidad innovadora de las medianas y grandes empresas nacionales, e incluso extranjeras, siempre bajo claros procesos de arbitraje social, y que por otra incluya las micro y pequeñas empresas como opciones eficientes para crear empleos, pero sobre todo que permita consolidar su desarrollo mediante capacitación, acceso al financiamiento y vinculación con empresas de mayor tamaño que ayuden a fortalecer su crecimiento y desarrollo. (Mungaray, Osuna, Ramírez, Ramírez, \& Escamilla, 2015)

En la última década ha ido tomando fuerza la idea de que el ámbito regional es más adecuado para el estudio de los sistemas de innovación en las empresas, dado que la región representa mayor homogeneidad desde el punto de vista cultural e institucional, lo cual implica mayor proximidad entre los empresarios, de los cuales ha adquirido gran importancia el subsistema empresarial. (Aceytuno \& Cáceres, 2012)

No debe dejar de considerar que el comprender el fenómeno de creación de nuevas empresas requiere tomar en cuenta también el enfoque sociocultural o institucional debido a que este es quien da las herramientas para entender el papel de los factores externos o del entorno. Es importante no dejar de lado el hecho de que la transmisión familiar del espíritu emprendedor ha generado una cuestión de gran interés, pues parece haberse confirmado la existencia de un efecto inverso que desalienta la creación de empresas en el caso de los descendientes directos de empresarios: las personas cuyos padres trabajan por cuenta propia se ven más renuentes a 
trabajar en su propia empresa y existe una mayor proporción, casi un $40 \%$, que muestra un mayor deseo para trabajar para la Administración Pública. Hay aspectos importantes al momento de crear una empresa propia, los cuales están relacionados con la posibilidad de poner en práctica las propias ideas, la posibilidad de crear algo propio y, asimismo, la independencia personal, entendida como posibilidad de trabajar con mayor libertad de decisión y de acción. Aspectos como la familia o status social, parecen tener una influencia muy reducida (Espí, Arana, Heras, \& Díaz, 2007).

Al referirse a los factores determinantes del emprendimiento empresarial relacionados con la influencia familiar, se puede mencionar el establecimiento de una red de contactos, una existencia previa de experiencia laboral, así como antecedentes empresariales en el grupo familiar, el liderazgo ejercido por el fundador de la empresa, la necesidad de logro del emprendedor y su familia, además se ha observado en las generaciones recientes, también a la formación técnica y profesional. (Santamaría, Moreno, Torrres, \& Cadrazco, 2013)

En las empresas familiares, la competencia y la lucha por un espacio en el mercado no frena sus posibilidades; esto representa nuevas oportunidades para los empresarios, ya que se ven retados a reconocer sus habilidades, salir de la informalidad y aplicar el protocolo y la sucesión familiar para llegar a ser más sólidas y poder enfrentar en el futuro nuevas estrategias (Arenas \& Rico, 2014). Se ha observado que en las empresas familiares, el hecho de que para muchos miembros su patrimonio está concentrado en la empresa y no salga de allí a corto plazo, ejerce una especial presión por cuidarlo e incrementarlo a largo plazo y de esa manera se entiende mucho mejor la diversificación de las nuevas empresas. (Nueno, 2011)

En la economía mundial son de gran importancia las micro, pequeñas y medianas empresas MIPYMES, ya que representan el mayor porcentaje de los negocios en los países. (Jiménez, Argueta, \& Espinoza, 2014)

\section{Problemática}

El emprendimiento de empresas es un factor de suma importancia dentro de la economía de un país, de ahí la importancia de fomentar la generación de pequeñas y medianas empresas, contribuyendo al desarrollo local, regional y nacional, donde la falta de empleo es un reto a vencer.

Un alto porcentaje de nuevas empresas permanece muy poco tiempo en operación, dando lugar a un alto índice de mortalidad y en consecuencia, un bajo crecimiento de empresas. En el estado de Chihuahua, al año 2013, se tenía un crecimiento negativo de empresas debido al alto nivel de mortalidad de las mismas a 37 meses de su creación. (INEGI, 2015) 
Dentro de la economía en México, las Micro, Pequeñas y Medianas Empresas (Mipymes) juegan un rol importante, se han llegado a considerar como la columna vertebral de la economía y del empleo nacional. Sin embargo, a pesar de su importancia, el promedio de vida de las Mipymes es de apenas dos años; lo cual se puede deber a consecuencia de factores internos ligados a los responsables de las empresas y a su competitividad. Es aquí donde las incubadoras de empresas pueden ser las canalizadoras de llevar a las Mipymes hacia una competitividad en el mercado y por consecuencia al éxito. (Salas, Valles, Galván, \& Cuevas, 2012)

\section{Justificación}

La generación de nuevas empresas constituye un eje de desarrollo dentro de cualquier país, es indispensable impulsar el crecimiento económico por medio de la creación de empresas, ligadas con la fuente de nuevos empleos. En México el número de emprendedores ha ido creciendo paulatinamente en los últimos años, principalmente en las micro, pequeñas y medianas empresas, lo cual es de gran peso como contribución al desarrollo del país. Es de gran importancia determinar cuáles son los factores que influyen en mayor medida en el hecho de emprender una empresa, a partir de donde se puedan formular estrategias que detonen la creación de nuevas empresas.

La Incubadora de Negocios FCA-UACH, como parte del Centro de Negocios, inició oficialmente en agosto de 2013, después de lograr la acreditación del Instituto Nacional del Emprendedor (INADEM) como parte de su red de incubadoras, logrando por segunda ocasión la reacreditación para el periodo 2014-2015, programa en el cual se brinda atención a los emprendedores que deseen poner su empresa y se atiende tanto a aquellos que llegan únicamente con una idea en su fase inicial hasta aquellos que tienen un negocio operando en temprana etapa, con la finalidad de lograr su formalización y consolidación en el mercado, esto se logra por medio de capacitación, asesoría en la elaboración de su plan de negocio y vinculación para la obtención de apoyos y financiamientos. Se ofrece el servicio a la comunidad en general, pero se tiene como prioridad la atención al mercado interno de alumnos y egresados de la FCA de la UACH.

El desarrollo local para la creación de empresas obedece a un concepto amplio que abarca múltiples funciones, entre las cuales se incluye la articulación de medidas dirigidas a la creación de empresas locales y a la animación social y económica tomando como referencia la potencialidad del territorio. En este sentido, los agentes de desarrollo local, que se integran en su mayoría en las instituciones propulsoras del este desarrollo, actúan como dinamizadores de las políticas implementadas por dichas instituciones, contribuyendo con su trabajo a la creación y posterior desarrollo de 
empresas, además de incidir en la sociedad local a través de la cultura y la movilización social. Estas funciones cobran relevancia en los momentos actuales de crisis económica, caracterizados por una fuerte destrucción de empleo y donde se hace necesario implementar acciones dirigidas a la reanimación de la actividad económica, además de incorporar nuevos valores tendentes a la sostenibilidad económica (Cantarero, Sanchis, \& Comeche, 2011).

\section{Materiales y Métodos}

Dentro de la Facultad de Contaduría y Administración (FCA) de la Universidad Autónoma de Chihuahua (UACH) en Chihuahua, México, opera un centro de apoyo para la generación de empresas, llamada Incubadora de negocios, cuya función es apoyar en la planeación e inicio de operaciones para las nuevas empresas, proporcionando servicios a la sociedad en general. Se analizó al total de la población registrada en la incubadora, con un total de 123 empresas, las cuales se formaron por iniciativa de una hasta 4 personas, todas son micro y pequeñas empresas.

El objetivo de la investigación fue determinar la relación entre el antecedente empresarial familiar y el emprendimiento de micro y pequeñas empresas. Se realizó un censo total de 123 empresas registradas en la Incubadora de Negocios de la FCA de la UACH. La investigación fue descriptiva y exploratoria; utilizando el método analítico-sintético, con un diseño transeccional, realizado en los meses de abril a junio de 2016. La variable dependiente a medir fue la generación de empresas y la variable independiente fue contar con antecedente empresarial familiar; los indicadores considerados fueron: operación actual de la empresa, sector económico de la empresa, género de los emprendedores, número de empleados de la empresa formada y sector económico de la empresa del familiar.

Se obtuvieron tablas de frecuencia, pruebas de chi cuadrada y análisis por conglomerados para las variables y sus indicadores.

\section{Resultados y discusión}

Del total de empresas registradas en la incubadora de negocios, la mayoría se encontraba en operación (53.3\%), como lo indica el cuadro 1; de las que no operaban, la mayoría estaban en proceso de establecimiento de operaciones. 
Cuadro 1. Empresas en Operación

\begin{tabular}{|c|c|c|c|c|c|}
\hline & Frecuencia & Porcentaje & Porcentaje válido & $\begin{array}{l}\text { Porcentaje } \\
\text { acumulado }\end{array}$ \\
\hline \multirow{3}{*}{ Válidos } & No & 56 & 45.5 & \multirow{5}{*}{$\begin{array}{c}46.7 \\
53.3 \\
100.0\end{array}$} & \multirow{5}{*}{$\begin{array}{c}46.7 \\
100.0\end{array}$} \\
\hline & $\mathrm{Si}$ & 64 & 52.0 & & \\
\hline & Total & 120 & 97.6 & & \\
\hline Perdidos & Sistema & 3 & 2.4 & & \\
\hline \multicolumn{2}{|c|}{ Total } & 123 & 100.0 & & \\
\hline
\end{tabular}

Un alto porcentaje de nuevas empresas permanece muy poco tiempo en operación, dando lugar a un alto índice de mortalidad y en consecuencia, un bajo crecimiento de empresas. En el estado de Chihuahua, al año 2013, se tenía un crecimiento negativo de empresas debido al alto nivel de mortalidad de las mismas a 37 meses de su creación. (INEGI, 2015)

Del total de emprendedores, el $58.5 \%$ respondió a la pregunta de contar con un familiar con una empresa, dentro de este porcentaje, la mayoría $(32.5 \%)$ de los emprendedores cuentan con un familiar que tiene una empresa, como se observa en el cuadro 2.

Cuadro 2. Familiar con una empresa

\begin{tabular}{|cc|c|c|c|c|}
\hline & & Frecuencia & Porcentaje & Porcentaje válido & $\begin{array}{c}\text { Porcentaje } \\
\text { acumulado }\end{array}$ \\
\hline \multirow{4}{*}{ Válidos } & No & 32 & 26.0 & 26.0 & 26.0 \\
& Si & 40 & 32.5 & 32.5 & 58.5 \\
& & 51 & 41.5 & 41.5 & 100.0 \\
& Total & 123 & 100.0 & 100.0 & \\
\hline
\end{tabular}

Cuadro 3. Parentesco con familiar empresario

\begin{tabular}{|cc|c|c|c|c|}
\hline & Frecuencia & Porcentaje & Porcentaje válido & $\begin{array}{c}\text { Porcentaje } \\
\text { acumulado }\end{array}$ \\
\hline \multirow{4}{*}{ Válidos } & Otro & 14 & 11.4 & 11.4 & 11.4 \\
& Abuelo & 1 & .8 & .8 & 12.2 \\
& Esposo (a) & 2 & 1.6 & 1.6 & 13.8 \\
& Hermano & 9 & 7.3 & 7.3 & 21.1 \\
& Padre & 14 & 11.4 & 11.4 & 32.5 \\
& & 83 & 67.5 & 67.5 & 100.0 \\
& Total & 123 & 100.0 & 100.0 & \\
\hline
\end{tabular}

El cuadro 3 muestra los porcentajes del parentesco existente entre el nuevo empresario y el familiar con una empresa, donde el 67.5\% manifestó algún parentesco con un familiar que cuenta con una empresa, del cual el $11.4 \%$ fue el porcentaje más alto que correspondió al padre y en la misma cantidad a otro parentesco no directo. 
De un 53.3\% de empresas que estaban en operación, el 26.7\% manifestó que su familiar tenía una empresa en operación, mientras que el $3.3 \%$ indicó lo contrario, el resto no respondió; lo cual se muestra en el cuadro 4. La relación se validó con la prueba Chi cuadrada, la cual presenta una alta significancia estadística, como se puede observar en el cuadro 5.

Cuadro 4. Relación entre empresa en operación del empresario y empresa del familiar en operación

\begin{tabular}{|c|c|c|c|c|c|c|}
\hline & \multicolumn{3}{|c|}{ Empresa del familiar operando? } & \multirow[t]{2}{*}{ Total } \\
\hline & & & & $\mathrm{Si}$ & No & \\
\hline \multirow{4}{*}{ Continúa operando? } & \multirow{2}{*}{ No } & Recuento & 53 & 2 & 1 & 56 \\
\hline & & $\%$ del total & $44.2 \%$ & $1.7 \%$ & $0.8 \%$ & $46.7 \%$ \\
\hline & \multirow{2}{*}{$\mathrm{Si}$} & Recuento & 28 & 32 & 4 & 64 \\
\hline & & $\%$ del total & $23.3 \%$ & $26.7 \%$ & $3.3 \%$ & $53.3 \%$ \\
\hline \multirow{2}{*}{ Total } & & Recuento & 81 & 34 & 5 & 120 \\
\hline & & $\%$ del total & $67.5 \%$ & $28.3 \%$ & $4.2 \%$ & $100.0 \%$ \\
\hline
\end{tabular}

Cuadro 5. Prueba Chi cuadrada entre nueva empresa operando y empresa de familiar operando

\begin{tabular}{|c|c|c|c|}
\hline & Valor & $\mathrm{gl}$ & $\begin{array}{c}\text { Sig. asintótica } \\
\text { (bilateral) }\end{array}$ \\
\hline Chi-cuadrado de Pearson & $35.612^{\mathrm{a}}$ & 2 & .000 \\
Razón de verosimilitudes & 41.158 & 2 & .000 \\
N de casos válidos & 120 & & \\
\hline
\end{tabular}

La mayoría de los nuevos empresarios que tenían una empresa en operación, manifestaron que su empresa no era del mismo giro que la empresa del familiar (24.2\% contra $6.7 \%$ ), lo cual se muestra en el cuadro 6. Al realizar la prueba Chi cuadrada entre estas dos variables, se obtuvo una alta significancia estadística, mostrada en el cuadro 7. Concordando lo anterior con lo que expresan Espí, Aran, Heras y Díaz (2007), los cuales indican que aspectos como la tradición familiar parecen tener una influencia muy reducida.

Cuadro 6. Relación entre empresa en operación y mismo giro de la empresa de familiar

\begin{tabular}{|ccc|c|c|c|c|}
\hline & & & \multicolumn{3}{|c|}{ Empresa del familiar mismo giro } & Total \\
& & & \multicolumn{3}{|c|}{ propio } & \\
\cline { 3 - 6 } & & & Si & No & \\
\hline & \multirow{2}{*}{ No } & Recuento & 53 & 1 & 2 & 56 \\
Continúa operando? & & $\%$ del total & $44.2 \%$ & $0.8 \%$ & $1.7 \%$ & $46.7 \%$ \\
& \multirow{2}{*}{$\mathrm{Si}$} & Recuento & 27 & 8 & 29 & 64 \\
& & $\%$ del total & $22.5 \%$ & $6.7 \%$ & $24.2 \%$ & $53.3 \%$ \\
Total & & Recuento & 80 & 9 & 31 & 120 \\
& & $\%$ del total & $66.7 \%$ & $7.5 \%$ & $25.8 \%$ & $100.0 \%$ \\
\hline
\end{tabular}


Cuadro 7. Prueba Chi cuadrada entre empresa en operación y mismo giro de la empresa de familiar

\begin{tabular}{|c|c|c|c|}
\hline & Valor & gl & $\begin{array}{c}\text { Sig. asintótica } \\
\text { (bilateral) }\end{array}$ \\
\hline Chi-cuadrado de Pearson & $37.042^{\mathrm{a}}$ & 2 & .000 \\
Razón de verosimilitudes & 42.413 & 2 & .000 \\
N de casos válidos & 120 & & \\
\hline
\end{tabular}

El cuadro 8 muestra la relación entre los nuevos empresarios y el parentesco con el familiar que tiene una empresa, donde se observa que las empresas que estaban en operación, el mayor porcentaje se da en otro familiar (no familiar directo) con un $10.8 \%$, muy similar al porcentaje con la relación con el padre (10\%). Al realizar una prueba Chi cuadrada entre estas dos variables, resultó estadísticamente significativa, como se muestra en el cuadro 9.

Cuadro 8. Relación entre empresa operando y parentesco con familiar empresario

\begin{tabular}{|c|c|c|c|c|c|c|c|c|}
\hline & \multicolumn{6}{|c|}{ Parentesco con familiar empresario } & \multirow[t]{2}{*}{ Total } \\
\hline & & & Padre & Hermano & $\begin{array}{l}\text { Esposo } \\
\text { (a) }\end{array}$ & Abuelo & Otro & \\
\hline \multirow{4}{*}{$\begin{array}{l}\text { Continúa } \\
\text { operando? }\end{array}$} & Recuento & 53 & 2 & 0 & 0 & 0 & 1 & 56 \\
\hline & $\begin{array}{c}\% \text { del } \\
\text { total }\end{array}$ & $44.2 \%$ & $1.7 \%$ & $0.0 \%$ & $0.0 \%$ & $0.0 \%$ & $0.8 \%$ & $46.7 \%$ \\
\hline & Recuento & 27 & 12 & 9 & 2 & 1 & 13 & 64 \\
\hline & $\begin{array}{c}\% \text { del } \\
\text { total }\end{array}$ & $22.5 \%$ & $10.0 \%$ & $7.5 \%$ & $1.7 \%$ & $0.8 \%$ & $10.8 \%$ & $53.3 \%$ \\
\hline \multirow[b]{2}{*}{ Total } & Recuento & 80 & 14 & 9 & 2 & 1 & 14 & 120 \\
\hline & $\begin{array}{c}\% \text { del } \\
\text { total }\end{array}$ & $66.7 \%$ & $11.7 \%$ & $7.5 \%$ & $1.7 \%$ & $0.8 \%$ & $11.7 \%$ & $100.0 \%$ \\
\hline
\end{tabular}

Cuadro 9. Prueba Chi cuadrada entre empresa operando y parentesco con familiar empresario

\begin{tabular}{|c|c|c|c|}
\hline & Valor & $\mathrm{gl}$ & $\begin{array}{c}\text { Sig. asintótica } \\
\text { (bilateral) }\end{array}$ \\
\hline Chi-cuadrado de Pearson & $37.512^{\mathrm{a}}$ & 5 & .000 \\
Razón de verosimilitudes & 44.835 & 5 & .000 \\
N de casos válidos & 120 & & \\
\hline
\end{tabular}

Con relación a lo anterior, coinciden Santamaría, Moreno, Torres y Cadrazco (2013), los cuales mencionan que dentro de los factores determinantes del emprendimiento empresarial relacionados con la influencia de la familia, se puede mencionar el uso del parentesco como mecanismo de subordinación y la necesidad de logro del emprendedor.

En el cuadro 10 se muestra la relación entre contar con un familiar empresario y el número de empleados del nuevo empresario, donde entre los que tienen un familiar con empresa, el porcentaje mayor (8.9\%) no cuentan 
con empleados, seguido de 2 empleados (5.7\%). Entre los que no tienen familiar con empresa el porcentaje mayor es de 3 empleados pero cero de $4 \mathrm{o}$ 5 empleados, donde si se presenta en el caso de quienes tienen familiar con empresa. Al realizar la prueba Chi cuadrada, se comprobó la relación entre las variables y se obtuvo una alta significancia estadística, como se muestra en el cuadro 11.

Cuadro 10. Relación entre contar con un familiar empresario y número de empleados en la nueva empresa

\begin{tabular}{|c|c|c|c|c|c|c|c|c|c|}
\hline & \multicolumn{8}{|c|}{ Cuántos empleados además de usted? } & \multirow[t]{2}{*}{ Total } \\
\hline & & Ninguno & 1 & 2 & 3 & 4 & 5 & Más de 5 & \\
\hline \multirow{6}{*}{$\begin{array}{l}\text { Familiar } \\
\text { con } \\
\text { empresa }\end{array}$} & 6 & 6 & 5 & 5 & 8 & 0 & 0 & 2 & 32 \\
\hline & $4.9 \%$ & $4.9 \%$ & $4.1 \%$ & $4.1 \%$ & $6.5 \%$ & $0.0 \%$ & $0.0 \%$ & $1.6 \%$ & $26.0 \%$ \\
\hline & 3 & 11 & 6 & 7 & 4 & 3 & 1 & 5 & 40 \\
\hline & $2.4 \%$ & $8.9 \%$ & $4.9 \%$ & $5.7 \%$ & $3.3 \%$ & $2.4 \%$ & $0.8 \%$ & $4.1 \%$ & $32.5 \%$ \\
\hline & 47 & 4 & 0 & 0 & 0 & 0 & 0 & 0 & 51 \\
\hline & $38.2 \%$ & $3.3 \%$ & $0.0 \%$ & $0.0 \%$ & $0.0 \%$ & $0.0 \%$ & $0.0 \%$ & $0.0 \%$ & $41.5 \%$ \\
\hline Recuento & 56 & 21 & 11 & 12 & 12 & 3 & 1 & 7 & 123 \\
\hline $\begin{array}{c}\% \text { del } \\
\text { total }\end{array}$ & $45.5 \%$ & $17.1 \%$ & $8.9 \%$ & $9.8 \%$ & $9.8 \%$ & $2.4 \%$ & $0.8 \%$ & $5.7 \%$ & $100.0 \%$ \\
\hline
\end{tabular}

Cuadro 11. Prueba Chi cuadrada entre contar con un familiar empresario y número de empleados en la nueva empresa

\begin{tabular}{|c|c|c|c|}
\hline & Valor & gl & $\begin{array}{c}\text { Sig. asintótica } \\
\text { (bilateral) }\end{array}$ \\
\hline Chi-cuadrado de Pearson & $90.687^{\mathrm{a}}$ & 14 & .000 \\
Razón de verosimilitudes & 107.365 & 14 & .000 \\
N de casos válidos & 123 & & \\
\hline
\end{tabular}

Se realizó un análisis entre la operación actual de la empresa y el número de empleados de la nueva empresa, obteniendo la medida de adecuación muestral de Kaiser- Meyer-Olkin (KMO), para demostrar la consistencia interna entre ambas variables, resultando significativo; además con la prueba de esfericidad de Bartlett, se obtuvo la Chi cuadrada que arrojó un valor significativo estadísticamente como se muestra en el cuadro 12.

Cuadro 12. KMO y Prueba de Bartlett para operación actual de la empresa y número de empleados en la nueva empresa

\begin{tabular}{|cc|c|}
\hline \multicolumn{2}{|c|}{ Medida de adecuación muestral de Kaiser-Meyer-Olkin. } & .500 \\
Prueba de esfericidad de Bartlett & Chi-cuadrado aproximado & 5.718 \\
& gl & 1 \\
& Sig. & .017 \\
\hline
\end{tabular}


Se realizó un conglomerado de K-medias, obteniendo 3 conglomerados finales, en el cual se muestra que 40 de 65 empresas en operación, cuentan con un familiar que tiene una empresa en el $61 \%$ de los casos, como se muestra en el cuadro 13 y cuadro 14.

Cuadro 13. Conglomerados de empresas en operación y familiar con empresa

\begin{tabular}{|c|c|c|c|}
\hline & \multicolumn{3}{|c|}{ Conglomerado } \\
\cline { 2 - 4 } & 1 & 2 & 3 \\
\hline Continúa operando? & 1 & 1 & 2 \\
Familiar con empresa & 1 & 2 & 2 \\
\hline
\end{tabular}

Cuadro 14. Número de casos por conglomerado de empresas en operación y familiar con empresa

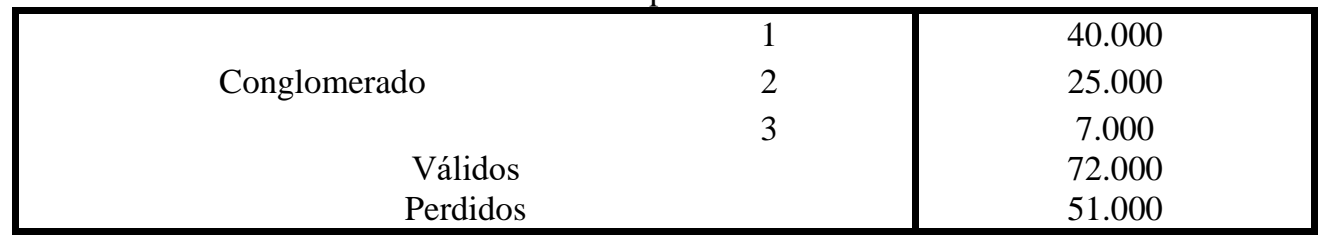

\section{Conclusión}

La principal conclusión del trabajo de investigación es que la mayoría de los emprendedores cuentan con un familiar con una empresa; respecto a los empresarios con una empresa operando, cuentan con un familiar que tiene una empresa. De quienes tienen un familiar con una empresa, lo más frecuente es que se trate del padre o de un familiar con parentesco no directo. La mitad de quienes tienen una empresa operando, tienen un familiar con una empresa en operación también.

La mayoría de los nuevos empresarios con una empresa en operación, manifestaron que su empresa no es del mismo giro que la empresa de su familiar con empresa. Los emprendedores que cuentan con una nueva empresa con un mayor número de empleados (4 o 5), se observa únicamente en quienes tienen un familiar con una empresa.

Sin embargo, de acuerdo con los resultados observados, no es determinante el hecho de no tener un familiar con una empresa, pudiendo influir también otros factores en el emprendimiento de nuevas empresas. Con base en lo cual se concluye de manera similar a lo que indican Pulgarín y Cardona (2011), que posiblemente el hecho de tener o no familiares cercanos, para determinar su incidencia en decidir ser empresario, parecería no influir en gran escala la definición del perfil como emprendedor.

Se recomienda continuar con un seguimiento a las empresas analizadas, para analizar a largo tiempo si el comportamiento de las empresas en operación sigue siendo diferente por el hecho de tener un familiar con una empresa. Es conveniente continuar con futuras 
investigaciones, a medida que el número de empresas registradas en la incubadora de negocios aumenten y vayan iniciando operaciones.

\section{References:}

1. Aceytuno, M. T., \& Cáceres, F. R. (2012). Los modelos europeos de transferencia de tecnología universidad-empresa. Revista de Economía Mundial, 215-238.

2. Arenas Cardona, H. A., \& Rico Balvín, D. (2014). La empresa familiar, el protocolo y la sucesión familiar. Estudios gerenciales, Vol. 30, Núm. 132, 252-258.

3. Cantarero S., S., Sanchis P., J., \& Comeche M., J. (2011). El papel del agente de desarrollo local en la creación de empresas en el contexto del desarrollo local. El caso valenciano. Scripta Nova. Revista Electrónica de Geografía y Ciencias Sociales.

4. Espí Guzmán, M. T., Arana Landín, G., Heras Saizarbitoria, I., \& Díaz de Junguitu González de Durana, A. (2007). Perfil emprendedor del alumnado universitario del campus de Gipuzkoa de la UPV/EHU. Revista de Dirección y de Administración de Empresas, Núm. 14, 83110.

5. INEGI. (9 de Febrero de 2015). Obtenido de Instituto Nacional de Estadística y Geografía: http://www3.inegi.org.mx/sistemas/temas

6. Jiménez Terrazas, C. P., Argueta López, G. V., \& Espinoza Granados, B. M. (2014). Revisión de literatura sobre mejora continua en MIPYMEs iberoamericanas. Nóesis, Vol. 23. Núm. 46, 140-162.

7. Mungaray L., A., Osuna M., J., Ramírez U., M., Ramírez A., N., \& Escamilla D., A. (2015). Emprendimientos de micro y pequeñas empresas mexicanas en un escenario local de crisis económica: El caso de Baja California, 2008-2011. Frontera Norte, 115-146.

8. Nueno, P. (2011). Iniciativa emprendedora y empresa familiar: emprendiendo a través de las generaciones. Universia Business Review, Núm. 32, 96-101.

9. Pulgarín, M. S., \& Cardona, A. M. (2011). Caracterización del comportamiento emprendedor para los estudiantes de administración de la Universidad de Rosario. Revista Escuela de Administración de Negocios, Núm. 71, 22-39.

10. Salas, J. F., Valles, E. K., Galván, A. K., \& Cuevas, T. (2012). Competitividad para las micro, pequeñas y medianas empresas en México, mediante las incubadoras de negocios. European Scientific Journal, Vol. 8, No. 25, 267-282.

11. Santamaría Escobar, Á. E., Moreno Arias, E. M., Torrres Torres, E. F., \& Cadrazco Parra, W. (2013). La influencia de la familia en el emprendimiento empresarial - El caso de tres pymes de los sectores 
comercial y de servicios de la ciudad de Sincelejo Sucre. Pensamiento Gerencial, Núm. 1.

12. Valdés Díaz de Villegas, J. A., \& Sánchez Soto, G. A. (2012). Las Mipymes en el contexto mundial: sus particularidades en México. Voces y contextos, Vol. VII, Núm. 14, 126-156. 